\title{
La Normalización Nacional e Internacional de cementos*
}

FRANCISCO SORIA SANTAMIRIA

Presidente de la cT-80 "Cementos y Cales" de IRANOR

\section{$R E S U M E N$}

Se hace una breve exposición de la situación $y$ tendencias de la industria cementiera para contemplar su incidencia en el desarrollo de la normativa de los conglomerantes.

Se comenta el desarrollo histórico de la normativa de cementos en España y la situación actual de las normas nacionales a nivel mundial.

Justificada la necesidad de una normativa internacional, se cita la participación de ISO $y$ otros Organismos, destacando y precisando la labor llevada a cabo por el Comité TC/51 del CEN (Comité Europeo de Normalización), para conseguir una norma europea sobre cementos.

\author{
$S U M M A R Y$
}

$A$ brief exposition of the situation and tendencies of the cement industry to contemplate its incidence in the development of norms of the conglomerates.

Comments are made on the historical development of the norms of cements in Spain and the present situation of the national norms at a world level.

Justifying the necessity of an international norm the participation of ISO and other Bodies is required, emphasizing the requirement of work to be carried out by the Committee TC/51 of the CEN (European Committee of Standardization), in order to achieve a European standard on cements.

\section{INTRODUCCION}

Antes de entrar en el tema objeto de esta exposición parece razonable hacer unos breves comentarios sobre la situación, evolución y tendencias de la industria cementera, para justificar el desarrollo de la normativa de los conglomerantes a todos los niveles.

Hoy en día, casi la mitad del costo de producción del cemento portland se debe al consumo de energía (34 \% la energía térmica y $10 \%$ la energía eléctrica), más del doble que el costo salarial $(19 \%)$.

Por ello, a lo largo de los últimos años, se han ido tomando ciertas medidas en el proceso de fabricación con tendencia, o bien a reducir el consumo de energía necesario para la cocción del clínker, o bien a utilizar otros combustibles más ventajosos.

Lo primero se ha conseguido con la introducción de intercambiadores de calor en vía seca (suspensión gaseosa) o semiseca (parrilla) y con la reducción gradual del proceso de vía húmeda. La inclusión de la precalcinación en la década de los 70, supuso un nuevo perfeccionamiento en esta tendencia.

En cuanto a lo segundo la solución adoptada ha sido, desde hace dos o tres años, la recon-

* Conferencia pronunciada en las II Jornadas Nacionales de Control de la Calidad en la Construcción sobre "Nuevos cementos", ceebradas en Madrid el 14 de Diciembre de 1982. 
versión masiva al carbón, la cual quedará prácticamente concluida en nuestro pais el presente año.

Estos factores han representado una reducción global del consumo de energía de 10-15 \% en los últimos 10 años.

A más largo plazo se preveen novedades en el terreno del ahorro energético, todavía en fase experimental, tratando las materias primas tradicionales $\mathrm{y} / \mathrm{u}$ otras procedentes de subproductos industriales con mineralizadores o en baño fundido, para conseguir cínkeres a más baja temperatura.

En lo que se relaciona con la tecnología de la fabricación, no debe olvidarse, finalmente, el deplorable balance energético que presenta el proceso de molienda.

Independientemente del proceso tecnológico en sí, otra posibilidad de ahorro de energía, más inmediata y más eficaz que las anteriores, es actuar sobre la composición del cemento, sustituyendo parte del clínker por otros productos con propiedades hidráulicas o capaces de desarrollarlas en presencia de los productos de hidratación del clínker (escorias siderúrgicas, puzolanas naturales o artificiales).

Debe señalarse, sin embargo, que estos cementos no son consecuenc:a o fruto de la crisis energética, pues se vienen produciendo con regularidad a lo largo de este siglo al tratar de preparar productos aptos para fabricar estructuras resistentes a ambientes de cierta agresividad (50 años de historia los siderúrgicos y 25 años los puzolánicos con cenizas volantes, aparte del empleo muchos años atrás de las puzolanas naturales).

Lo que sucede es que, ante la presión del coste de la caloría, la producción de estos cementos, ya viejos, adquiere gran actualidad y, por ello, el tema de las adiciones en general (escorias, puzolanas y filler) es objeto de especial consideración por parte de la normativa actual, tanto a nivel nacional como internacional.

\section{HISTORIA DE LA NORMATIVA DE CEMENTOS A NIVEL NACIONAL}

\subsection{Normas}

El Instituto Nacional de Racionalización y Normalización se creó en 1949, bajo la denominación de Instituto de Racionalización del Trabajo.

En él se crean muy pronto dos Comisiones relacionadas con el tema de cementos: la CT-7 "Ensayo de Materiales" y la CT-41 "Construcción". En la primera se normalizan métodos de ensayo y en la segunda especificaciones.

Con anterioridad a 1960 se publicaron tres normas de ensayo basadas en la NELC (Normas de Ensayo Laboratorio Central) y en la ASTM (concretamente, R.I. en cementos puzolánicos, calor de hidratación y medida de la superficie específica por permeabilímetro Blaine).

Entre 1963 y 1966 se publicaron siete normas de Ensayos y once de Nomenclatura y Definiciones, basadas en el PCCH-61 y 64 .

Desde entonces no se volvieron a publicar más normas sobre cementos en IRANOR.

En 1977 se crea la Comisión CT-80 "Cementos, cales y yesos", que en abril de 1981 se desglosa en dos por razones de homogeneidad y equiparación a otras Comisiones extranjeras: la CT-80, con la denominación de "Cementos y cales" y la CT-102, con la denominación 
de "Yeso y productos del yeso". Esto está en consonancia con las correspondientes ISO TC-74 y TC-152.

Con la creación de esta nueva Comisión CT-80 se pretende revitalizar, potenciar y actualizar con más rapidez la normativa del sector, independientemente de la actividad de las Comisiones 7 y 41 que se desenvuelven en un campo de actividades mucho más amplio.

La necesidad de su puesta en marcha, por otro lado, se justifica, entre otros, por los siguiente motivos:

- Para seguir de cerca las directrices de la futura e inmediata normativa europea con la que se marcha en coordinación desde tiempo atrás,

- para recoger y encauzar el impacto creado por el tema del ahorro energético y sus consecuencias en la tipología de cementos.

- y, finalmente, dada su constitución con personas de todos los ámbitos interesados, para canalizar, coordinar y contemplar cuantas sugerencias sirvan para aclarar ideas en el triángulo fabricante-consumidor-Administracción.

Las tareas de la Comisión se desarrollan a través de cuatro Grupos de Trabajo:

- GT-1: Ensayos físicos y mecánicos.

- GT-2: Ensayos químicos.

- GT-3: Definiciones, nomenclatura y especificaciones.

- GT-4: Toma de muestras y control de calidad.

Hasta la fecha, en estos Grupos, se han preparado cuatro borradores de norma UNE: dos de ensayos químicos, uno de ensayos físicos y otro de ensayos mecánicos. Para su elaboración se han manejado como documentos de base los correspondientes aprobados en voto preliminar en la TC-51 del CEN (Comité Europeo de Normalización), cumplimentados, en algunos casos, con campañas de ensayos sobre aspectos puntuales que exigían alguna aclaración.

El IRANOR, como miembro del CEN, delega en esta Comisión su participación en la discusión y elaboración de la Norma Europea, trabajando activamente en dicha Comisión TC-51 y en cuatro de sus ocho Grupos de Trabajo.

\subsection{Pliegos de Condiciones}

España se ha regido a través de toda su historia, en lo que a normativa de cementos se refiere, por Ordenes o Decretos Ministeriales, emanados sucesivamente en el tiempo del Ministerio de Fomento, del Ministerio de Obras Públicas o de la Comisión Interministerial de más reciente creación.

La primera Orden Ministerial surge en Noviembre de 1924, en la que simplemente se indica la "obligación de señalar las características del cemento en los Pliegos de Condiciones para la celebración de subastas".

En Febrero de 1930 se publica por el Ministerio de Fomento el Primer "Pliego General de Condiciones para la recepción de los Aglomerantes Hidráulicos".

Tras varias Ordenes Ministeriales alusivas a modificaciones no sustanciales del Pliego anterior, surge en Enero de 1960 el nuevo "Pliego General de Condiciones para la recepción 
de Conglomerantes Hidráulicos", muy distinto del anterior, y que recoge como idea sustancial la última innovación de las Recomendaciones ISO sobre el ensayo de resistencias mecánicas, que mencionaremos más adelante.

Rápidamente surge el Pliego de 1964, con la denominación del "Pliego General de Condiciones para la recepción de Conglomerantes Hidráulicos en Obras de carácter Oficial".

Entre las características principales de este Pliego caben destacar las siguientes: elevación de las categorías resistentes en todos los tipos de cemento, creación de los cementos de adición, introducción del permeabilímetro Blaine simultáneamente con el tamiz como índice de la finura y ampliación del ensayo de expansión en autoclave a todos los cementos.

Finalmente, a partir de 1974, y por disposición gubernamental, se comenzó la revisión del PCCH-64 a cargo de una Comisión Interministerial y de un Grupo de Trabajo, revisión que concluyó en el actual "Pliego de Prescripciones Técnicas Generales para la Recepción de Cementos", con ámbito de aplicación a nivel nacional. Es lo que se llama el RC-75.

La novedad más trascendente de este Pliego la constituye la introducción de los cementos portland con adiciones activas, acompañada de la inclusión de los puzolánicos con cenizas volantes y los compuestos y la supresión de los denominados cementos portland comerciales.

Siguiendo la línea de Pliegos anteriores y habida cuenta de los progresos en la fabricación y de la evolución de otras Normas, se suprime, en varios tipos y clases de cemento, la categoría resistente 250 y se crea una categoría nueva 550 en los portland y portland con adiciones activas, a la vez que surgen categorías superiores en los cementos siderúrgicos y puzolánicos.

También aparece un grupo de cementos, paradójicamente sólo portland en algunos casos, con propiedades adicionales: resistencia inicial (ARI), resistencia al yeso (PY), bajo calor de hidratación (BC) y blancura (B).

Se suprime el ensayo de permeabilidad Blaine y queda sólo la limitación del residuo máximo sobre el tamiz de 4.900 mallas $/ \mathrm{cm}^{2}$.

En el ensayo de expansión se introducen las agujas de Le Chatelier y se suprime el ensayo en autoclave en todos los cementos, salvo para los portland puros.

Tras algunas modificaciones de tipo químico, como limitar la magnesia sólo en los cementos portland, se introduce como novedad un método para medir el color de los cementos blancos, estableciendo la correspondiente especificación.

Este Pliego, por otro lado, es más explícito que los anteriores en lo que se refiere a toma de muestras y envasado e identificación de los cementos.

Como comentario adicional diré que el RC-75 presenta 33 cementos diferentes, algunos sólo posibles sobre el papel, como ocurre, por ejemplo, con el P-350 ARI.

\section{ESTADO ACTUAL DE LAS NORMATIVAS NACIONALES A NIVEL MUNDIAL}

En la última edición de las "Normas de Cemento en el Mundo", edita por el CEMBUREAU en Mayo de 1980, existían 68 países con Normas propias y 26 que adoptaban una Norma extranjera (10 la AFNOR, 12 la BS y 4 la ASTM). 
No obstante, de los 68 países con Norma propia, en lo que respecta al ensayo de resistencias mecánicas, 31 han adoptado el ensayo ISO-RILEM-CEMBUREAU (de ellos 24 europeos), 18 el ensayo de la BS (singularmente en Africa y Asia) y 11 el ensayo de la ASTM (7 en América y 4 en Asia). De los restantes con Norma propia cabe destacar Rusia, Japón y Brasil, que se encuentran en la línea del método ISO con ligeras divergencias.

A grandes rasgos, en lo que concierne a nomenclatura, definiciones y, sobre todo, a especificaciones, se mantiene la influencia del país cuya norma se toma de base, reduciéndose las tendencias a dos grandes grupos: grupo europeo continental, por una parte, y un grupo que reune la influencia inglesa y americana, por la otra.

Las diferencias esenciales pueden centrarse en los siguientes puntos:

1. En las Normas ASTM y BS sólo existían especificaciones para los cementos portland hasta hace relativamente pocos años. Concretamente la ASTM hizo una norma única para los cementos de mezcla en 1967.

2. Las limitaciones en parámetros químicos, como el residuo insoluble y la pérdida por calcinación, son más restrictivas en las normas angloamericanas que en las europeas, donde incluso se han llegado a suprimir en algunos países (Francia, por ejemplo).

3. Las normas inglesas y americanas relacionan el contenido de $\mathrm{SO}_{3}$ con la finura y el contenido de $\mathrm{C}_{3} \mathrm{~A}$ del clínker. En Europa se establece un contenido de $\mathrm{SO}_{3}$ fijo, variando en algunos casos con el tipo de cemento.

4. En la ASTM se manejan las limitaciones químicas basadas en la composición potencial del clínker, para definir los cinco tipos de cemento portland. En Europa sólo se hace uso de ello para definir los equivalentes al tipo $\mathrm{V}$ (resistentes a sulfatos).

5. Las normas BS y ASTM valoran la finura, preferencialmente, por Blaine. En Europa se maneja más el residuo sobre un tamiz o, incluso, se suprime la especificación (caso de Francia, por ejemplo). Debe señalarse que Francia, en contrapartida, establece un ensayo de retracción, único país en el mundo que normaliza esta propiedad.

6. Las normas europeas e inglesa fijan la medida de la expansión con las agujas de Le Chatelier, en tanto que la ASTM establece en exclusiva el ensayo de autoclave, ensayo que según palabras de su Pres:dente, requiere una urgente revisión.

7. En Europa se insiste especialmente en el comportamiento mecánico del cemento, singularmente en el esfuerzo a compresión, que sirve de base para dominarlo y clasificarlo. Con el mismo fin, la ASTM se rige más por parámetros de tipo químico.

8. Tanto en las BS como en la ASTM se garantiza la calidad a los usuarios por ensayos de control de recepción. En Francia y otros países europeos, la marca de calidad evita los controles de recepción, apoyándose en un control practicamente continuo de la fabricación y un control frecuente de expediciones. Se hacen intervenir, en varios países, los conceptos de límite superior y límite inferior en resistencias, garantizando un valor medio y una regularidad del producto. Es de esperar que también la ASTM establezca también limitaciones en este sentido, en breve plazo.

\section{NECESIDAD DE UNA NORMALIZACION INTERNACIONAL DE CEMENTOS}

El cemento fue uno de los primeros materiales de construcción objeto de reglamentación o normalización. 
Lógicamente, esta normativa comenzó a nivel nacional y las primeras reglas para fijar la calidad del producto se editaron, hace más de un siglo, a nivel interno en las propias fábricas. Estos documentos industriales fueron los que se manejaron más tarde por las Administraciones Públicas para hacer sus Reglamentos o Pliegos de Condiciones, en primera fase, y por los Organismos Nacionales de Normalización después, para hacer las Normas. Así surgieron, a lo largo de este siglo, las Normas DIN, BS, AFNOR, ASTM, etc.

La iniciativa de las fábricas respondía a una necesidad de crearse una imagen de "marca de calidad", al tratarse de un producto nacido del tratamiento, más o menos empírico, de rocas y tierras, en estado natural primero (cementos naturales) y por mezclas convenientemente dosificadas después (cementos artificiales) y conseguido en Empresas reducidas, casi de ámbito familiar, pero en franca expansión a raiz del descubrimiento del cemento portland artificial.

La instauración de un sistema de control de calidad y el establecimiento de una marca data ya desde hace más de 50 años, ante la importancia que llegaron a adquirir, hacia los años 20, las exportaciones de cementos europeos a otros continentes y la necesidad de mantener un prestigio.

Acabada la segunda guerra mundial surge la explosión en la producción de cemento y continua en aumento el tonelaje de exportación por parte de la Europa Occidental, llegando a los 30 millones de toneladas en el presente año, de los cuales el $40 \%$ corresponde a España, primer exportador mundial en estos momentos.

Precisamente, para facilitar y potenciar estas exportaciones, la industria europea del cemento comenzó a considerar, hacia los años 50, la necesidad de iniciar trabajos que condujesen a una normalización internacional para unificar, al menos en principio, lo concerniente a los métodos de ensayo.

El cemento no es como el acero, producto sobre el cual se pueden medir directamente sus características esenciales. Para medir la resistencia mecánica del cemento, producto que rara vez se emplea solo, se prepara un mortero que se moldea, se compacta, se cura un cierto tiempo y, finalmente, se somete a rotura.

Este mortero, a su vez, fija su arena con una cierta granulometría, las proporciones de cemento, arena y agua, su método de compactación, la forma y dimensiones de las probetas y sus condiciones de curado. Con tantas variables, los posibles valores numéricos de la resistencia resultan totalmente dispares.

La puesta a punto de un método internacional para medir la resistencia de los cementos se comenzó precisamente en el seno de la industria cementera europea (CEMBUREAU), más tarde en colaboración con la RILEM (Reunión Internacional de Laboratorios de Ensayo de Materiales) y, finalmente, en el seno del Comité TC-74 ISO "Cementos y Cales", para acabar en una Recomendación de este Organismo a finales de 1960. Este método fue afortunado pues, como ya hemos dicho, se aplica hoy día prácticamente en toda Europa y algunos países de otros Continentes.

\section{ORGANISMOS INTERNACIONALES DE NORMALIZACION}

\subsection{ISO (International Standardization Organization)}

En 1946, justamente recién acabada la II Guerra Mundial, se crea en Londres la ISO, con la colaboración de 25 países. 
Su objetivo principal es promocionar la normalización mundial para facilitar los intercambios de mercancias y servicios entre países, a la vez que intentar conseguir un entendimiento mutuo en el campo intelectual, científico, técnico y económico.

Actualmente reside en Ginebra y está formada por 71 Comités Miembros Nacionales a través de su Organismo Nacional de Normalización y por 16 Miembros Correspondientes. Estos últimos suelen ser Organismos de la Administración de países en vías de desarrollo que no tienen todavía su propio Organismo o Instituto de Normalización. España èstá representada en ISO desde 1951 a través de su Instituto de Rac:onalización y Normalización.

Sus actividades se desarrollan a través de Comités Técnicos, con Subcomités y Grupos de Trabajo que se ocupan de los distintos aspectos encomendados a cada Comité.

Las normas ISO no tienen carácter obligatorio y van destinadas a los Organismos Nacionales, que las emplean como base para la elaboración de su propia normativa.

Uno de los 160 Comités que funcionaban recientemente es, precisamente el TC-74 "Cementos y cales".

Aparte de la Recomendación ya citada sobre "Método de ensayo mecánico de cementos. Resistencia a la compresión y a la flexión del mortero plástico (ISO/R 679), este Comité publicó entre los años 67 y 68, las siguientes Recomendaciones sobre cemento:

ISO/R 597: Definiciones y terminología de cementos.

ISO/R 680: Análisis químico de cementos. Componentes principales del cemento portland.

ISO/R 681: Análisis químico de cementos. Componentes menores del cemento portland.

ISO/R 682: Análisis químico de cementos. Determinación de azufre en estado de sulfuro.

ISO/R 683: Ensayo de puzolanicidad para el control de los cementos puzolánicos.

En 1969, la Comunidad Económica Europea emitió una directriz tratando de suprimir las barreras al comercio de productos entre los Estados miembros. Entre estos productos se encontraba el cemento.

La industria cementera consideró que, aunque no existian barreras propiamente dichas en su caso, sería positivo, para facilitar los intercambios, unificar y armonizar los métodos de ensayo, la definición y nomenclatura de cementos y, finalmente, los procedimientos de control de calidad y de conformidad a normas.

A propuesta de un país Miembro de la Comunidad, el CEN (Comité Europeo de Normalización), fundado en 1961 con residencia en Bruselas, creó el Comité Técnico TC-51 "Cementos" que inició sus actividades en 1973 y del que hablaremos con más detalle más adelante.

Como el Secretariado del CEN/TC 51 y el de ISO/TC 74 es el mismo (Instituto Belga de Normalización), se esperaba que el primero, que empezó su tarea revisando las seis Recomendaciones ISO preexistentes, haría propuestas de revisión en breve plazo, pero esto no ocurrió así.

Hacia 1974, cuando el Secretariado del ISO/TC 74 quiso reanudar las actividades del Comité, encontró poco interés por parte de los miembros que no forman parte del CEN y fue frenado por los que eran miembros, para evitar duplic:dad de tareas.

Ante un apremio por parte de países como Japón, Canadá y Sudáfrica por la paralización 
de las actividades de la ISO desde hace tantos años, la División técnica n. 3 propuso al TC 74 preparar unas propuestas de normas ISO, sin esperar el final de los trabajos de CEN.

En esta línea se han preparado y redactado dos textos, que están precisamente a la espera del voto final en el TC 51 de CEN. Estos documentos son:

74 N 229: Métodos de ensayo de cementos. Determinación de las resistencias mecánicas.

74 N 230: Métodos de ensayo de cementos. Determinación del tiempo de fraguado y de la estabilidad.

El primero de ellos puede considerarse como una propuesta de revisión de ISO/R 679, mientras que el segundo es una nueva propuesta.

En breve plazo se espera redactar una propuesta de Revisión de ISO/R 681 y 682 . Análisis de cementos.

\subsection{CEN (Comité Europeo de Normalización)}

Como se ha dicho anteriormente, el CEN se creó en 1961 y en 1973 comenzó sus tareas el TC-51 "Cementos".

Pueden ser miembros del CEN todos los Organismos Nacionales de Normalización de los países miembros de la CEE o de la AELE o susceptibles de serlo. España pertenece desde 1974. Actualmente tiene 17 miembros (toda la Europa Occidental).

Sus objetivos se resumen en tres:

- armonizar las normas establecidas en los países miembros y elaborar una norma europea.

- apoyar a la normalización mundial en el seno de la ISO.

- prever los servicios de certificación sobre la base de normas europeas.

Lo mismo que en la ISO, sus actividades se desarrollan a través de Comités, Subcomités y Grupos de Trabajo.

A diferencia de la ISO, un voto positivo en el CEN significa el compromiso de otorgar la categoría de norma nacional al documento aceptado, dentro de un plazo que podía ser de hasta dos años, es decir, la obligación de publicarlo como Norma Nacional. Por el contrario, un voto negativo o una abstención, supone libertad absoluta para decidir entre su publicación o la conservación de cualquier norma nacional. También sería posible que si los Ministros de la Comunidad lo aprueban como Directriz sea obligatoria su aceptación, con voto positivo o negativo.

En el CEN/TC-51 "Cementos", se crearon, en principio, cinco grupos de Trabajo, referentes todos ellos a ensayos:

GT-1: Ensayos mecánicos.

GT-2: Ensayos físicos.

GT-3: Ensayos químicos.

GT-4: Determinación del contenido de adiciones.

GT-5: Ensayo de puzolanicidad. 
Al año siguiente (1974) se creó el Grupo 6 "Definiciones y Terminología", posteriormente el Grupo 7 "Toma de muestras" y, a finales de 1980, el GT-8 "Especificaciones". En la última reunión celebrada en Roma hace unos días, se ha creado un nuevo grupo para tratar de "Procedimientos de conformidad", en conexión con los grupos 6 y 8.

En los nueve años que lleva funcionando esta Comisión se han producido documentos muy completos en los primeros grupos. La tarea es lenta porque hay que tener en cuenta que, en ocasiones, ha sido necesario desarrollar una tarea experimental para definir un nuevo método de ensayo o modificar otro preexistente.

De estos primeros Grupos están a la espera del voto final, después de haber emitido el voto preliminar, los siguientes documentos:

Grupo 1. prEN 158: Ensayo de resistencia mecánica.

Grupo 2. prEN 112: Ensayo de fraguado y estabilidad.

Grupo 3. prEN 75: Análisis químico.

Grupo 4. prEN 114: Determinación del contenido de escoria.

Grupo 5. prEN 196: Ensayo de puzolanicidad.

Están en fase de preparación proyectos de norma complementarios al prEN 75 (Determinación de ion cloro y $\mathrm{CO}_{2}$ ), un proyecto que englobe los ensayos de determinación del contenido de clínker, escoria, ceniza volante y puzolana del GT-4 y otro a cargo del GT-7 sobre "Toma de muestras".

El Grupo 6 ha elaborado un cuadro de cementos (Tabla I) último de una serie de proyectos y que quizás no sea definitivo. Establece, en principio, cuatro tipos de cemento:

- el tipo I para cemento portland, con la posible incorporación de hasta $5 \%$ de adición (escoria, ceniza, puzolana o filler).

- el tipo II, en el que se establecen, en principio, tres grupos (portland siderúrgico, portland puzolánico y portland compuesto). En todos ellos el contenido mínimo de clínker es de $65 \%$ y el resto adición, pudiendo incluirse hasta $5 \%$ de filler, en todos los casos.

- el tipo III para cementos siderúrgicos con una proporción de escoria siderúrgica de horno alto comprendida entre 36 y $80 \%$ y la posibilidad de añadir hasta $5 \%$ de filler.

- el tipo IV para cementos puzolánicos, con un contenido máximo de $40 \%$ de puzolana. No admite la incorporación de filler.

Dentro de este Grupo 6 se está desarrollando desde hace más de un año un programa de trabajo en 15 laboratorios de 10 países para ensayar 6 cementos: un portland $(\mathrm{P})$, un puzolánica (PZ), un siderúrgico (BLF) y tres cementos portland con adición de filler calizo en proporciones del orden de 11,27 y $11,5 \%$, respectivamente $\left(F_{1}, F_{2}\right.$ y $\left.F_{3}\right)$.

Aparte de los ensayos físicos, químicos y mecánicos, según Norma, se han programado otros, en forma de mortero y hormigón, dirigidos a estudiar su comportamiento en cuanto a carbonatación, resistencia al hielo y corrosión de armaduras.

Los resultados de que se dispone hasta la fecha, proporcionan la siguiente información:

- Las exigencias de agua de los cementos con filler, a igualdad de consistencia en hormigones, es del mismo orden que las del resto de los cementos con los que se comparan. 
- Los hormigones preparados con el cemento $F_{1}$ presentan mal comportamiento a los ciclos hielo-deshielo. El resto de los hormigones queda englobado en un huso común, con el siguiente orden de preferencia:

$$
\mathrm{BLF}>F_{3} \simeq \mathrm{PZ}>\mathrm{P}>F_{2}
$$

- El huso se estrecha al reducir la relación agua/cemento, con notable mejoría en el comportamiento global de todos los hormigones, salvo el preparado con el cemento $F_{1}$.

- La mayor profundidad de carbonatación tuvo lugar en los morteros preparados con el cemento $F_{1}$ y la más reducida en el mortero del cemento $F_{2}$. El resto de los cementos se sitúa en posiciones intermedias.

Una primera conclusión de estas experiencias es que, en el caso de la presencia de filler calizo, en principio, tienen gran importancia las características y propiedades de dicho filler.

El grupo 7 "Toma de muestras" ha presentado un nuevo borrador de propuesta de norma en la pasada reunión de Roma que, quizás, se someta a voto preliminar.

En cuanto al grupo 8 "Especificaciones", último que se puso en marcha hace dos años, desarrolló, en una primera fase, una recopilación de propiedades definidas y delimitadas en las distintas normas de los países miembros, haciendo sólo referencia a las alusivas a los tipos de cemento presentados por el grupo 6 y que mencionamos anteriormente (no se tienen en cuenta, ni cementos como el aluminoso, de albañilería o sobresulfatado, ni cementos con propiedades especiales como la resistencia a sulfatos o el calor de hidratación). Posteriormente, se discutieron en Grupo y Comisión las distintas delimitaciones, para acabar presentando el cuadro adjunto (Tabla II) en la reciente reunión de Roma.

\section{TAB L A I}

Tablas de tipos de cemento

\begin{tabular}{|c|c|c|c|c|c|c|}
\hline \multirow[b]{2}{*}{ Tipo } & \multirow[b]{2}{*}{ Designación } & \multicolumn{5}{|c|}{ Proporción en masa $\%$} \\
\hline & & Clínker & $\begin{array}{l}\text { Escoria } \\
\text { granulada } \\
\text { de horno } \\
\text { alto }\end{array}$ & Puzolana & $\begin{array}{l}\text { Ceniza } \\
\text { volante }\end{array}$ & Fíller \\
\hline I & Cemento portland & $95 \ldots 100$ & \multicolumn{4}{|c|}{$0 \ldots 5$} \\
\hline II-S & Cementos portland con escoria & $65 \ldots 90$ & $10 \ldots 35$ & \multicolumn{3}{|c|}{$0 \ldots 5$} \\
\hline II-Z & $\begin{array}{l}\text { Cemento portland con puzo- } \\
\text { lana }\end{array}$ & $65 \ldots 90$ & $0 \ldots 5$ (1) & & $10 \ldots 35$ & $0 \ldots 5 \quad(1)$ \\
\hline $\mathrm{II}-\mathrm{S} / \mathrm{Z}$ & $\begin{array}{l}\text { Cemento portland con escoria } \\
\text { y puzolana }\end{array}$ & $65 \ldots 88$ & $6 \ldots 29$ & & $6 \ldots 29$ & $0 \ldots 5$ \\
\hline III & Cemento de horno alto & $20 \ldots 64$ & $36 \ldots 80$ & \multicolumn{3}{|c|}{$0 \ldots 5$} \\
\hline IV & Cemento puzolánico (3) & $\geqslant 60$ & $0 \ldots 5(1)$ & $<$ & 0 & $0 \ldots 5(1)$ \\
\hline
\end{tabular}

(Los números de la tabla se refieren a la base del cemento, excluyendo sulfato cálcico y aditivos).

(1) El contenido total de escoria de horno alto y filler no excederá el $5 \%$ en masa.

(2) Las proporciones de escoria granulada de horno alto que excederá del $65 \%$ se señalará adicionalmente.

(3) El cemento puzolánico cumplirá los requisitos del ensayo de puzolanicidad de acuerdo con CEN. 
T A B L A II

Propuesta preliminar de WG 8-TC 51/CEN

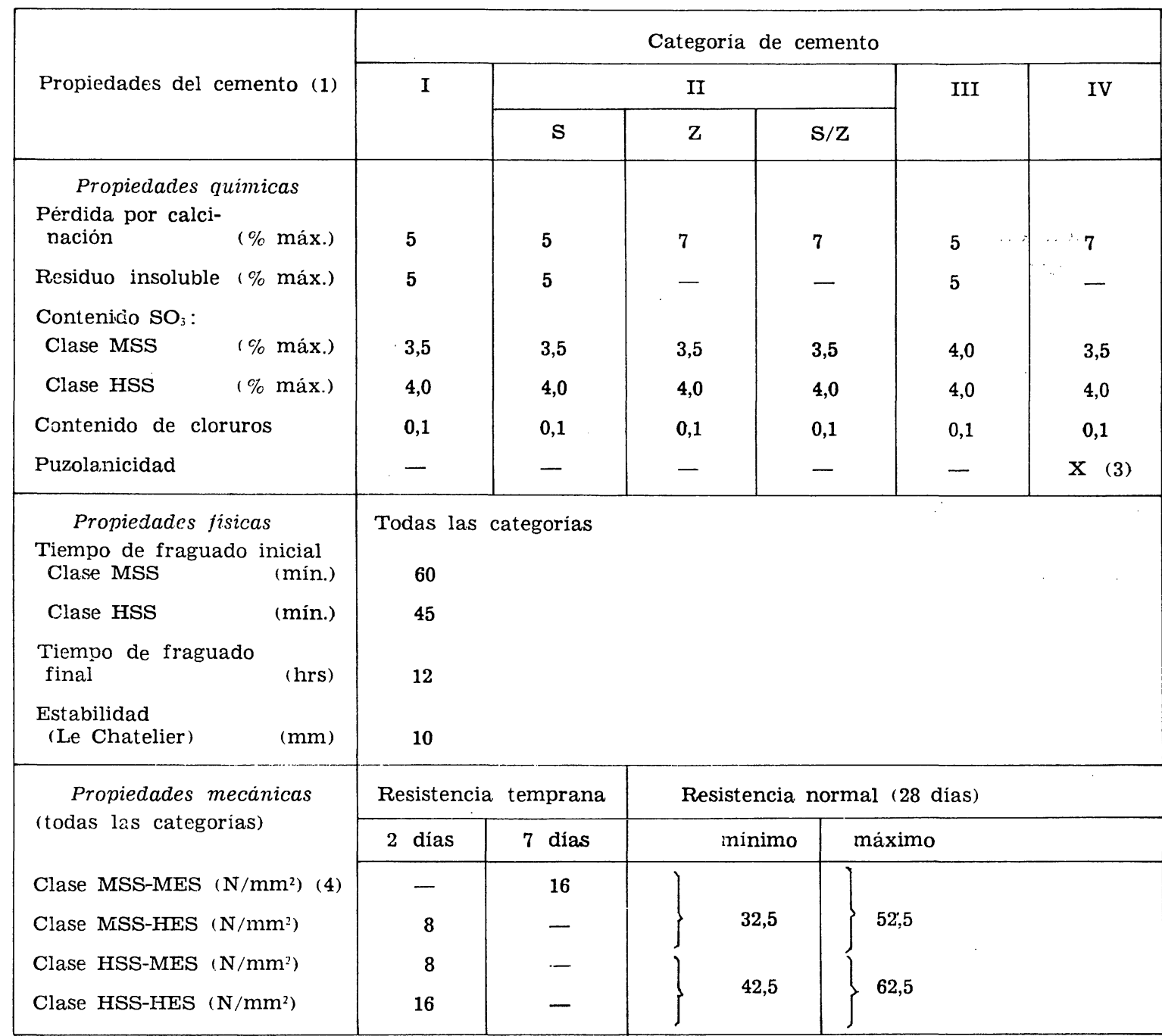

(1) Todos los límites son absolutos.

(2) Para cementos con un contenido de cloruros más elevado debe señalarse el hecho.

(3) Ensayo de puzolanicidad.

(4) MSS $=$ resistencia normal media.

HSS $=$ resistencia normal alta

MES = resistencia inicial media.

HES $=$ resistencia inicial alta.

Los grupos 6 y 8 están muy relacionados entre sí y cuidan y discuten mucho sus limitaciones: uno debe denominar y definir y el otro fijar prestaciones.

España tiene representante, en estos momentos, en los grupos 4, 6, 7 y 8 y asiste con regularidad alguna representación de la CT-80 a las reuniones de la Comisión.

Se encuentran presentes en la Comisión TC-51 y en alguno de sus grupos de trabajo, aparte de los representantes de Centros de Investigación y fábricas de cemento, representantes de ERMCO (Asociación Europea de Hormigón Preamasado), BIBM (Asociación Internacional de Hormigón Prefabricado) y FIEC (Federación Internacional de la Construcción). 


\subsection{Otros Organismos Internacionales de Normalización}

A nivel internacional existen otros Organismos cuyo ámbito se restringe a ciertas áreas o zonas de la Tierra, como ocurre con el CEN.

Así, se encuentra la COPANT (Comisión Panamericana de Normas Técnicas), fundada en 1961, con 15 miembros titulares, entre los que se encuentran Estados Unidos, Méjico y la mayor parte de los países sudamericanos. Todos ellos están representados a través de sus respectivos Institutos de Normalización.

También, en 1966 se fundó la ASAC (Comité Consultivo de Normas Asiáticas), que engloba prácticamente a todos los países asiáticos, incluyendo Australia.

Más recientemente (1967) se creó la ASMO (Organización Arabe de Normalización y Metrología), cuyos miembros son la mayoría de los países árabes del Próximo Oriente.

El objetivo o la política de todos ellos es hacer el mayor uso posible de las normas ISO (de la que suelen ser miembros), con las modificaciones o adiciones estrictamente necesarias para que respondan a las necesidades de sus respectivas Comunidades.

Como resumen de esta rápida exposición se concluye que el gran Organismo Internacional es la ISO que, con la colaboración de unos 2.000 Organismos Técn:cos y más de 100.000 especialistas del mundo entero, ha publicado ya unas 5.000 normas, en sus tres lenguas oficiales (inglés, francés y ruso).

\section{CONSIDERACIONES FINALES}

En una encuesta llevada a cabo por la Agencia Internacional de la Energía de la OCDE. acerca de la evolución de la composición de los cementos hasta el año 2.000, se llega a la conclusión de que, en este momento, los cementos portland compuestos, en la Europa Occidental, representan el $50 \%$ de su producción y que, progresivamente, supondrán hasta el $80 \%$, relegando los cementos portland puros a cementos especiales para emplear en casos muy particulares, que exijan prestaciones excepcionales.

Así se llegará a fin de siglo con un aumento de producción del orden del $20 \%$ con relación a la actual y un consumo energético un $20 \%$ inferior al de hoy día.

No hay duda que la tendencia al ahorro energético, por una parte, y al reciclado de subproductos industriales por razones ecológicas, por la otra, conducen al empleo masivo de materiales, de los que se precisa que cumplan un mínimo de cualidades para garantizar las prestaciones de los cementos en el futuro.

Esto obliga a desarrollar una política de concierto entre los Organismos Oficiales y los sectores industriales implicados, tanto en la producción de estos materiales (yacimientos, siderúrgia y centrales térmicas) como en la fabricación del cemento. Su misión será establecer los niveles de calidad y conseguir un producto final regular y de calidad adecuada que garantice la preparación de hormigones duraderos y estables.

Con este fin, a la industria cementera le compete la preparación, en exclusiva, de estos cementos, ya que existen una serie de condiciones que sólo se pueden cumplir con los equipos e instalaciones de que se dispone en sus fábricas.

En primer lugar, las fábricas tratan y homogeneizan grandes cantidades de material a gra- 
nel y en polvo y tienen posibilidades correctoras o de ajuste, cosa que no es posible en una hormigonera.

En segundo término, los controles en la fábrica son continuos e, incluso, se complementan en laboratorios externos si hay sello de conformidad, cosa que no puede hacerse en obra por la colocación inmediata del producto de la hormigonera.

Finalmente, hacer un cemento compuesto no es simplemente mezclar dos cosas: deben de estudiarse previamente los componentes y las condiciones óptimas de mezcla, en cada caso, en función de la naturaleza y características fisicoquímicas de aquellos (molturabilidad, influencia en la reología de pastas, retención de agua, retracción plástica, etc., etc.).

El usuario, por su parte, debe de tener una buena información y conocimiento de las posibilidades de uso de los cementos compuestos, disponiendo de las indicaciones de uso adecuadas para los múltiples casos en que no es imprescindible el cemento portland.

Volviendo a la Normalización, de unos años a esta parte, no sólo la industria sino los propios Gobiernos han empezado a considerar que los Organismos Internacionales como CEN e ISO son esenciales para preparar Normas que puedan ser aplicadas o mencionadas en las leyes y Reglamentos.

Los países en vías de desarrollo, aún cuando sean miembros de Organismos como la ISO, no contribuyen prácticamente en su funcionamiento, pero sí tienen interés en el fruto de su trabajo porque lo consideran el lenguaje idóneo para encauzar la transferencia de tecnología.

Existe ya el GATT (Acuerdo General de Aduanas y Comercio) que supone un intento para eliminar, o al menos mitigar, las barreras técnicas al comercio internacional, causadas por diferencias entre leyes, reglamentos, pliegos y normas, a nivel nacional.

Para llevar a cabo su función este Organismo considera a la Normalización internacional como el vehículo, la herramienta, para armonizar la legislación o normativa nacionales, apremiando a los Organismos Internacionales de Normalización en su tarea y recomendando que, en aquellos casos en que se requiera una norma o reglamento y existieran normas internacionales apropiadas, los Gobiernos u Organismos interesados las usen como base para dichos Reglamentos o Normas.

De aquí se deduce la necesidad de que los expertos de las Administraciones Públicas participen en la normalización nacional y sigan muy de cerca los trabajos que se desarrollan a nivel internacional.

A su vez, la adaptación de la normativa nacional a la internacional, prestigia aquélla y hace que sus normas se tengan más en consideración en los Reglamentos Técnicos.

Un segundo aspecto de la normalización es la certificación.

Los sistemas de certificcación a nivel nacional son diferentes, lo mismo que las normas; esto crea muchos problemas a la hora de la exportación, con la agravante de que nuestros exportadores se ven obligados a obtener todo tipo de certificaciones cuando acuden a un mercado exterior; en contrapartida, en España, en ocasiones, pueden entrar los mismos productos sin ningún tipo de trabas al no existir ningún sistema de certificación que exigir.

Los sistemas que existen, aparte de ser incompletos y escasos, surgen de forma anárquica 
promovidos por diversos Organismos Públicos y Privados, sin coordinación entre ellos y empleando como documentos de base distintas Normativas.

En este sentido, paralelamente a los Organismos de Normalización ISO y CEN, ya citados, se han creado los respectivos Organismos Internacionales de certificación: CENCER (Comité de Certificación del CEN) y CERTICO (Comité de Certificación de ISO), en los que España está representada a través de IRANOR.

Repito, y para terminar, los Institutos de Normalización a todos los niveles son el marco ideal para desarrollar todas estas tareas. Es el lugar donde, en una atmósfera cordial, todas las partes interesadas - fabricantes, usuarios, Administración y laboratorios de ensayo y control- están representadas y pueden deliberar con toda libertad.

\section{BIBLIOGRAFIA}

(1) J. Calleja: "Las nuevas normas españolas para cemento" Materiales de Construcción, n..$^{\circ}$ 164, 4. trim. 1976.

(2) P. K. Mefta: “Cement Standards. Evolution and Trends". Amer. Soc. Test. Mat. STP 993.1978.

(3) Cement Standards of the World. Cembureau. 1980.

(4) C. Cerquella: "Normalización europea. Normalización internacional". Boletín de la Normalización española, n. ${ }^{\circ} 7-8$, julio-agosto 1980.

(5) P. Dutron: "La respuesta de la industria cementera a los problemas económicos y técnicos actuales" Materiales de Construcción, n. $184,4 .^{\circ}$ trimestre 1981.

(6) “Necesidades y dificultades de una normalización europea de cementos". Ciments et Chaux, enero 1982.

(7) Compte Rendu de la Journée du CERILH 1982". Ciments, Betons, Plâtres. Rev. Mat. n. 735 , febr. 1982.

(8) “Los cementos al filler: productos de calidad, economía de energía”. Ciments et Chaux, marzo 1982.

(9) Olle StuRen: "Desarrollo de la Normalización Internacional y su importancia en el Comercio Exterior". Boletín de la Normalización Española, n. 5, Septiembre 1982.

(10) Documentación CEN TC-51.

\section{publicación del i.e.t.c.c.}

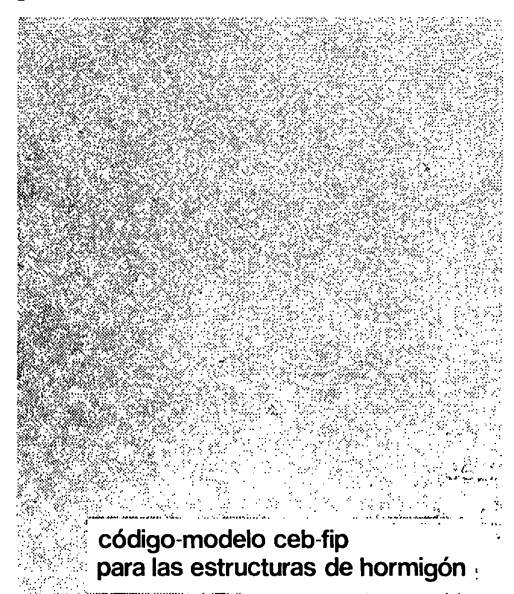

El Instituto Eduardo Torroja, miembro activo tanto del Comité Eurointernacional del Hormigón (CEB), como de la Federación Internacional del Pretensado (FIP), ha tomado a su cargo la traducción y edición de esta importante normativa.

Aunque presentado con el titulo de "Código Modelo CEB/FIP 1978" este documento incorpora los dos primeros volúmenes de este "Sistema Unificado Internacional de Reglamentación Técnica de Ingenieria Civil». El prime volumen de este "Sistema Unificado" es el denominado "Reglas comunes Unificadas para los diferentes tipos de obras y materiales", donde se exponen los criterios y formatos de seguridad a que han de ajustarse los diferentes de maderal, que han de configurar la totalidad del antedicho sistema.

El segundo volumen es propiamente el Código Modelo para las Estructuras de Hormigón. Fruto de la colaboración de dos asociaciones del prestigio del CEB y la FIP, desde mediados de los 60 , incorpora los avances cientificos y tecnológicos producidos en los últimos años sin detrimento alguno de la claridad y operatividad que deben presidir un código que pretende ser, ante todo, un auxiliar práctico para los técnicos de la construcción.

El Código sigue en su estructura las reglas más o menos clásicas: una primera parte dedicada a los datos Cones para el ćlculo (propiedades de los materiales, datos relativos a prena pare tolerancias): en segundo lugar se presentan las reglas de proyecto estructural lacciones, solicitaciones, estados limites iltimos y de utilización, reglas de detalle para el armadol; y, por último, ejecución, mantenimiento y control de calidad.

También incluye reglas para estructuras con elementos prefabricados y estructuras de hormigón con áridos ligeros. Los Anejos del Código se refieren a: terminologia, proyecto mediante la experimentación, resistencia al fuego, tecnologia del hormigón, comportamiento en el tiempo del hormigón y fatiga.

Un volumen encuadernado en cartoné, de $21 \times 30 \mathrm{~cm}$, compuesto de 340 páginas, Madrid, mayo 1982

Precios España 2.500 ptas. Extranjero 50 \$USA 